\title{
Dieta de Callithrix penicillata (E. Geoffroy, 1812) (Primates, Callitrichidae) introduzidos na Ilha de Santa Catarina
}

\author{
Luciana Zago ${ }^{1 *}$ \\ João M. D. Miranda ${ }^{1,2}$ \\ Cássio Daltrini Neto ${ }^{3}$ \\ Cristina V. Santos ${ }^{4}$ \\ Fernando C. Passos ${ }^{1}$ \\ ' Laboratório de Biodiversidade, Conservação e Ecologia de Animais Silvestres \\ Departamento de Zoologia, Universidade Federal do Paraná \\ Caixa Postal 19020, CEP 81531-980, Curitiba - PR, Brasil \\ ${ }^{2}$ Universidade Estadual do Centro-Oeste, Guarapuava - PR, Brasil \\ ${ }^{3}$ Laboratório de Restauração Ambiental Sistêmica, Departamento de Botânica \\ Universidade Federal de Santa Catarina, Florianópolis - SC, Brasil \\ ${ }^{4}$ Laboratório de Educação Cerebral, Departamento de Psicologia \\ Universidade Federal de Santa Catarina \\ * Autor para correspondência \\ luazagos@gmail.com
}

Submetido em $11 / 10 / 2012$

Aceito para publicação em 19/03/2013

\section{Resumo}

Espécies do gênero Callithrix foram introduzidas em diferentes regiões do Brasil e suas adaptações alimentares podem ser fatores que possibilitam seus estabelecimentos nesses ambientes. Este estudo objetiva determinar atividades alimentares e recursos vegetais utilizados na dieta de Callithrix penicillata introduzidos em um fragmento florestal urbano na Ilha de Santa Catarina. No Parque Ecológico do Córrego Grande (PECG) foram monitorados três grupos de C. penicillata em duas fases de seis meses. Os itens alimentares consumidos foram registrados em amostragens mensais de um dia para dois grupos e dois dias para outro grupo. Para o grupo amostrado por dois dias mensais também foram registradas as atividades alimentares. A atividade predominante foi Presa Animal com 70\% dos registros, seguida por Exsudato, com 16\%, Fruto, com $8 \%$ e Suplementar, com 5\%. A família Fabaceae foi mais expressiva para exploração de Exsudato e Myrtaceae, para Fruto. Os resultados encontrados são semelhantes aos de distribuições originais de Callithrix. Dentre essas semelhanças foram encontradas espécies vegetais registradas em suas dietas. A disponibilidade desses vegetais associada à preferência de $C$. penicillata por hábitats fragmentados e a disponibilidade de alimentos fornecidos por seres humanos são fatores que parecem possibilitar o estabelecimento dessa espécie no PECG.

Palavras-chave: Fragmento florestal urbano; Ilha de Santa Catarina; Mico-estrela

\section{Abstract}

Diet of Callithrix penicillata (E. Geoffroy, 1812) (Primates, Callitrichidae) introduced to the Santa Catarina Island. Species of the genus Callithrix were introduced to different Brazilian regions and their dietary adaptations may be factors which allow them to settle in these environments. This study aims to determine 
feeding activities and plant resources used in the diet of Callithrix penicillata introduced to an urban forest patch in the Santa Catarina Island. At the Corrego Grande Ecological Park (PECG), three C. penicillata groups were monitored in two phases for six months. The food items eaten were recorded in 1 day monthly samples for 2 groups and in 2 days for another group. Regarding the group sampled for 2 days a month, the feeding activities were also recorded. The predominant activity was Animal Prey, with $70 \%$ of records, followed by Exudate, with $16 \%$, Fruit, with $8 \%$, and Supplemental, with $5 \%$. The Fabaceae family was the most significant one with regard to the exploitation of Exudate and the Myrtaceae, concerning Fruit. The results found are similar to those of original distributions of Callithrix. Among these similarities, one found plant species recorded in their diet. The availability of these plants associated to the $C$. penicillata preference for patched habitats and the availability of food provided by human beings are factors which seem to allow these species to settle at PECG.

Key words: Black-tufted-ear marmoset; Santa Catarina Island;Urban forest patch

\section{Introdução}

O gênero Callithrix (Erxleben, 1777) (Primates: Callitrichidae) é composto por espécies com tamanho corporal reduzido (300 a 450 g) e adaptados à vida arbórea (HERSHKOVITZ, 1977). As exigências metabólicas desse pequeno tamanho corpóreo relacionam-se a ocupação de um nicho insetívoro, onde invertebrados suprem necessidades proteicas que frutos, comuns na dieta de primatas do Novo Mundo, não podem suprir (MARROIG; CHEVERUD, 2005). Além de presas animais como artrópodes e pequenos vertebrados, estes primatas também se alimentam de frutos e exsudatos (HERSHKOVITZ, 1977).

A habilidade em consumir exsudatos é devida a adaptações morfológicas dentárias e do trato digestivo que os permitem escavar troncos de árvores e consumir e digerir a goma então exsudada (HERSHKOVITZ, 1977; RYLANDS; FARIA, 1993). Essa habilidade em explorar exsudatos, ricos em carboidratos e disponíveis em todas as estações, permite que mesmo em épocas de escassez de outros recursos alimentares as necessidades energéticas desses primatas sejam supridas (FERRARI, 1993; CASTRO; ARAÚJO, 2006). Essa vantagem adaptativa está entre os fatores que levam as espécies mais exsudatívoras (com maior especialização dentária para a exsudativoria) da família Callitrichidae, $C$. penicillata (E. Geoffroy, 1812) e C. jacchus (Linnaeus, 1758) (STEVENSON; RYLANDS, 1988), a ter grupos maiores e mais estáveis, maiores taxas reprodutivas e áreas de vida menores que as de outras espécies menos exsudatívoras (RYLANDS, 1996).

Essas características ecológicas podem estar entre os fatores que possibilitam que espécies mais exsudatívoras (C. penicillata e C. jacchus) ocupem até mesmo com grandes densidades populacionais: habitats fragmentados, ambientes urbanos e habitats fora de suas distribuições naturais (FERRARI, 2009). Com tamanha flexibilidade ecológica, estas espécies não estão ameaçadas de extinção (CHIARELLO et al., 2008). Já $C$. flaviceps (Thomas, 1903) e C. aurita (Humboldt, 1812), espécies menos exsudatívoras do gênero Callithrix (com especializações dentárias menos desenvolvidas para a exsudativoria) (STEVENSON; RYLANDS, 1988), são espécies com menor flexibilidade ecológica (FERRARI, 1993; MARTINS; SETZ, 2000), estando ambas ameaçadas de extinção (C. aurita como "Vulnerável" e C. flaviceps como "Em Perigo") (CHIARELLO et al., 2008). As principais ameaças a estas espécies menos flexíveis são a perda e descaracterização do habitat, além da hibridização e competição com espécies congêneres (C. penicillata e C. jacchus) introduzidas em seus habitats (CHIARELLO et al., 2008; FERRARI, 2009).

A espécie C. penicillata possui distribuição natural predominante no bioma Cerrado, mas também ocorre na Caatinga e na Mata Atlântica, com limite sul no estado de São Paulo (VIVO, 1991; RYLANDS et al., 1993). Já C. jacchus distribui-se predominante no bioma Caatinga, mas também ocorre na Mata Atlântica e em transições de Caatinga e Cerrado, com limite sul no litoral do estado da Bahia (VIVO, 1991; RYLANDS et al., 1993). Enquanto C. geoffroyi (Humboldt, 1812) distribui-se predominante no bioma Mata Atlântica, mas também ocorre em transições com Cerrado, com limite sul no estado de Minas Gerais (VIVO, 1991; RYLANDS et al., 1993). Entretanto, introduções destas espécies vêm ocorrendo em diferentes regiões do Brasil (COIMBRA-FILHO, 
1990; VIVO, 1991), incluindo a Ilha de Santa Catarina, onde há cerca de 30 anos foram introduzidas essas três espécies (SANTOS et al., 2007). Diante disso, este trabalho objetiva determinar as atividades alimentares e os recursos vegetais utilizados na dieta de Callithrix penicillata introduzidos em um fragmento urbano de forma a possibilitar a compreensão dos fatores que permitem o estabelecimento desta espécie neste hábitat.

\section{Material e Métodos}

A coleta de dados foi realizada no Parque Ecológico do Córrego Grande (PECG), um fragmento urbano de 21,5 ha de Floresta Ombrófila Densa em estágios iniciais e médios de regeneração. O PECG está localizado na Ilha de Santa Catarina $\left(27^{\circ} 35^{\prime} \mathrm{S}, 48^{\circ} 30^{\prime} \mathrm{W}\right)$, onde três grupos de C. penicillata foram monitorados e identificados pelas siglas: GL, GG e GP. Os dois primeiros grupos eram compostos por oito indivíduos, enquanto o último era composto por 12 indivíduos ao início do estudo (Tabela 1).

O período de amostragem foi dividido em duas fases: fase I, de Outubro de 2008 a Março de 2009 e fase II, de Abril de 2009 a Setembro de 2009. Em cada fase foi monitorado um grupo principal com amostragens mensais de dois dias completos (considerou-se um dia completo o período entre a hora em que se encontrou o grupo até a mesma hora do dia seguinte), enquanto os outros grupos foram monitorados com amostragens de um dia completo por mês. Na fase I, o grupo principal foi GL, enquanto na fase II, devido a mudanças nas áreas de vida de GL que dificultaram suas amostragens, o grupo principal foi GG (Tabela 1).

TABELA 1: Composição dos grupos GL, GG e GP [categorias etárias propostas por Yamamoto (1993)] e número de dias por mês em esforços amostrais no período de outubro de 2008 a setembro de 2009 (células vazias representam meses em que os esforços amostrais foram insuficientes para se determinar as composições dos grupos com exatidão).

\begin{tabular}{|c|c|c|c|c|c|c|c|c|c|c|c|c|}
\hline Mês & Out & Nov & Dez & Jan & Fev & Mar & Abr & Mai & Jun & Jul & Ago & Set \\
\hline GRUPO GL & \multicolumn{6}{|c|}{ Fase I } & \multicolumn{6}{|c|}{ Fase II } \\
\hline Amost. (dias/mês) & 2 & 2 & 2 & 2 & 2 & 2 & 2 & 1 & 1 & 1 & 1 & 1 \\
\hline Adultos & 5 & 5 & 4 & 4 & 4 & 4 & 4 & & & & & \\
\hline Subadultos & 0 & 0 & 0 & 3 & 1 & 1 & 1 & & & & & \\
\hline Juvenis & 3 & 3 & 3 & 0 & 0 & 0 & 0 & & & & & \\
\hline Infantes & 0 & 0 & 0 & 0 & 0 & 0 & 0 & & & & & \\
\hline TOTAL & 8 & 8 & 7 & 7 & 5 & 5 & 5 & & & & & \\
\hline GRUPO GG & \multicolumn{6}{|c|}{ Fase I } & \multicolumn{6}{|c|}{ Fase II } \\
\hline Amost. (dias/mês) & 1 & 1 & 1 & 1 & 1 & 1 & 2 & 2 & 2 & 2 & 2 & 2 \\
\hline Adultos & & & 6 & 6 & 6 & 6 & 5 & 5 & 6 & 5 & 5 & 5 \\
\hline Subadultos & & & 0 & 2 & 2 & 2 & 1 & 1 & 0 & 0 & 0 & 0 \\
\hline Juvenis & & & 2 & 0 & 0 & 0 & 0 & 0 & 0 & 3 & 3 & 3 \\
\hline Infantes & & & 0 & 0 & 3 & 3 & 3 & 3 & 3 & 0 & 0 & 2 \\
\hline TOTAL & & & 8 & 8 & 11 & 11 & 9 & 9 & 9 & 8 & 8 & 10 \\
\hline GRUPO GP & \multicolumn{6}{|c|}{ Fase I } & \multicolumn{6}{|c|}{ Fase II } \\
\hline Amost. (dias/mês) & 1 & 1 & 1 & 1 & 1 & 1 & 1 & 1 & 1 & 1 & 1 & 1 \\
\hline Adultos & & & & 7 & 7 & 7 & 7 & & 5 & & 7 & \\
\hline Subadultos & & & & 0 & 2 & 2 & 2 & & 2 & & 0 & \\
\hline Juvenis & & & & 2 & 0 & 0 & 2 & & 2 & & 3 & \\
\hline Infantes & & & & 3 & 3 & 3 & 1 & & 1 & & 0 & \\
\hline TOTAL & & & & 12 & 12 & 12 & 12 & & 10 & & 10 & \\
\hline
\end{tabular}


Para todos os grupos amostrados foram registrados, pelo método de varredura instantânea com anotação pontual em intervalos de 10 minutos (ALTMANN, 1974), os itens vegetais consumidos (frutos ou exsudatos) cujas árvores foram marcadas e posteriormente identificadas. Pelo mesmo método também foram registradas as atividades alimentares dos grupos principais. Essas atividades alimentares foram organizadas em categorias onde se registrou tanto o ato de consumir o alimento quanto a procura e esforços para obtenção do mesmo (forrageio). Essas categorias de alimentação/forrageio foram: Presa Animal (artrópodes, pequenos vertebrados, entre outros); Fruto; Exsudato (gomas exsudadas através da escavação feita pelos saguis em troncos vegetais); e Suplementar (alimentos fornecidos por humanos, visitantes ou vizinhos do parque, em sua maioria frutas).

\section{Resultados}

$\mathrm{Na}$ fase I, a atividade alimentar predominante do grupo GL foi Presa Animal com 76\% $(\mathrm{n}=980)$ dos registros, seguida por Exsudato com $14 \%(n=184)$,
Fruto com 5\% $(\mathrm{n}=63)$ e Suplementar com 5\% $(\mathrm{n}=$ 62). Na fase II, manteve-se o padrão de utilização dos recursos alimentares, com a porcentagem dos registros do grupo GG para Presa Animal obtendo 64\% $(n=787)$ dos registros; Exsudato, 19\% $(\mathrm{n}=229)$; Fruto, 12\% (n $=144)$; e Suplementar, 6\% $(\mathrm{n}=74)($ Figura 1$)$.

Foram determinadas 25 diferentes espécies exploradas pelos mico-estrelas nas categorias alimentares Fruto (14) e Exsudato (11). Estas espécies pertencem a 13 diferentes famílias vegetais e estavam distribuídas em um total de 80 árvores, das quais 43 foram utilizadas para o consumo de frutos e 37 para a exploração de exsudatos (Tabela 2). A família Fabaceae foi a mais expressiva para exploração de exsudatos, tanto em número de espécies $(\mathrm{n}=7)$ como em registros de uso $(61,9 \%)$. As espécies Inga marginata, Acacia mearnsii e Acacia podalyriifolia foram as mais exploradas no consumo de exsudatos (Tabela 2). Já para o consumo de frutos, a família mais expressiva foi Myrtaceae, tanto em número de espécies $(\mathrm{n}=5)$ como em número de registros $(19,6 \%)$, sendo Syzygium jambolanum, Musa paradisiaca e Morus nigra as espécies mais exploradas (Tabela 2).

FIGURA 1: Porcentagem de registros para o grupo GL, na fase I (Total = 1289 registros), e para o grupo GG, na fase II (Total = 1234 registros), das categorias de alimentação/forrageio: Presa Animal, Exsudato, Fruto e Suplementar.

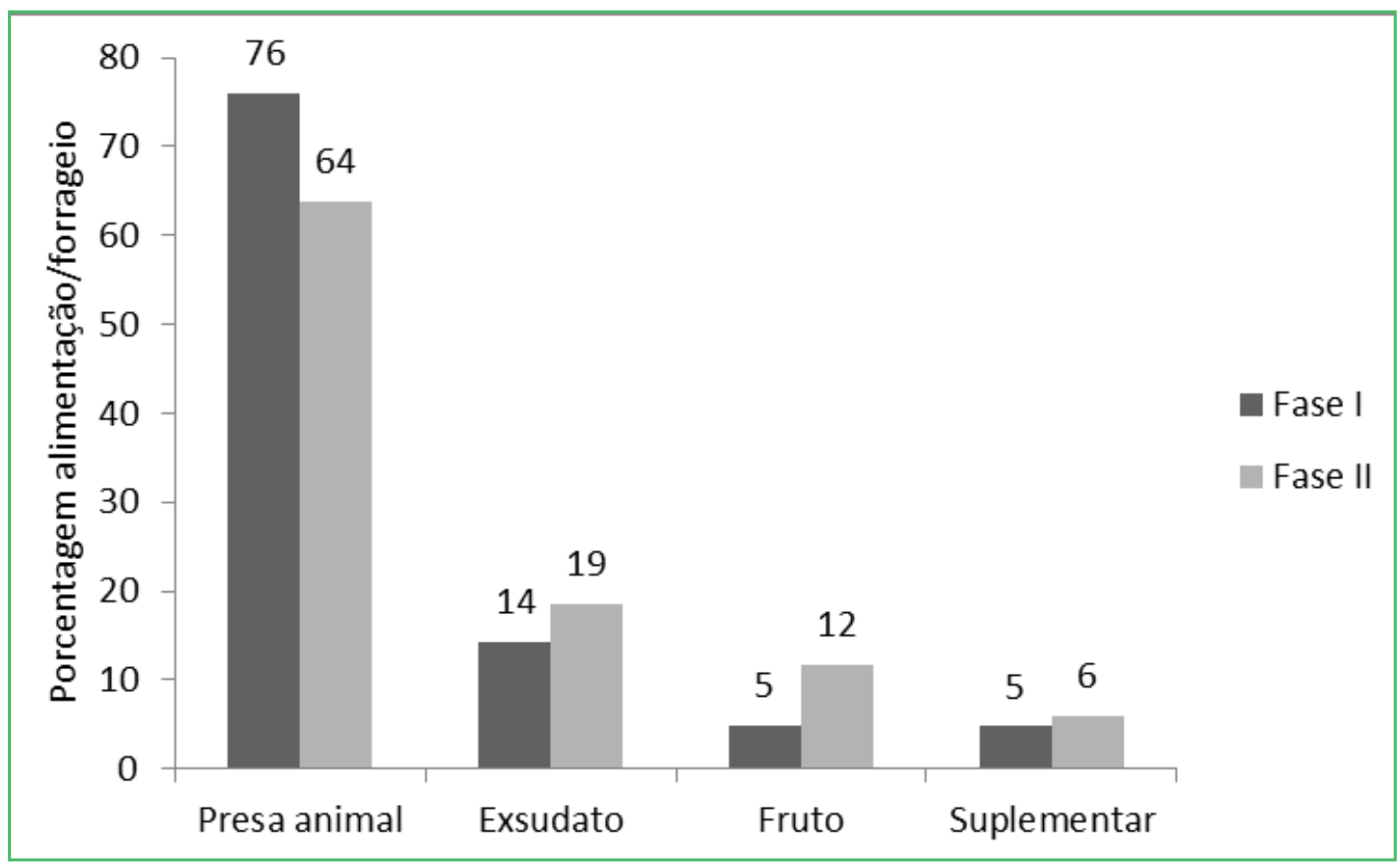


TABELA 2: Espécies vegetais exploradas por Callithrix penicillata no PECG, seus respectivos números de indivíduos explorados (Ind.), itens explorados $(\mathrm{F}=$ fruto, $\mathrm{E}=$ exsudato), frequência de explorações (\%) e referências bibliográficas que citam a utilização destas espécies (R. Sp.) ou gêneros (R. Gn.) vegetais por Callithrix spp.

\begin{tabular}{|c|c|c|c|c|c|}
\hline Família / Espécie & Ind. & Item & $\%$ & R. Sp. & R. Gn. \\
\hline \multicolumn{6}{|l|}{ Annonaceae } \\
\hline Annona mucosa Jacq. & 1 & $\mathrm{~F}$ & 1 & - & $\mathrm{j}, \mathrm{m}$ \\
\hline \multicolumn{6}{|l|}{ Asteraceae } \\
\hline Mikania vitifolia DC. & 1 & $\mathrm{E}$ & 0,7 & - & - \\
\hline \multicolumn{6}{|l|}{ Urticaceae } \\
\hline Cecropia glaziovii Snethl. & 2 & $\mathrm{~F}$ & 0,3 & - & $\mathrm{a}, \mathrm{f}, \mathrm{j}$ \\
\hline \multicolumn{6}{|l|}{ Malvaceae } \\
\hline Chorisia speciosa A.St.-Hil. & 3 & $\mathrm{E}$ & 0,3 & - & - \\
\hline \multicolumn{6}{|l|}{ Combretaceae } \\
\hline Terminalia catappa $\mathrm{L}$. & 1 & $\mathrm{E}$ & 0,2 & $\mathrm{~d}, \mathrm{e}, \mathrm{j}$ & $\mathrm{c}$ \\
\hline \multicolumn{6}{|l|}{ Euphorbiaceae } \\
\hline Aleurites moluccana $($ L.) Willd & 1 & $\mathrm{E}$ & 0,2 & $\mathrm{~h}$ & - \\
\hline \multicolumn{6}{|l|}{ Fabaceae } \\
\hline Acacia mearnsii De Wild. & 6 & $\mathrm{E}$ & 8,9 & - & $b, e, f$ \\
\hline Acacia podalyriifolia A. Cunn. ex G. Don & 4 & $\mathrm{E}$ & 8,4 & - & $b, e, f$ \\
\hline Caesalpinia peltophoroides Benth. & 3 & $\mathrm{E}$ & 0,2 & - & $\mathrm{K}$ \\
\hline Enterolobium contortisiliquum (Vell.) Morong & 1 & $\mathrm{E}$ & 7,2 & $\mathrm{~d}, \mathrm{j}$ & $c, g, i$ \\
\hline Inga marginata Willd. & 5 & $\mathrm{E}$ & 31,8 & $\mathrm{~h}, \mathrm{l}$ & $\mathrm{e}, \mathrm{f}, \mathrm{g}, \mathrm{i}, \mathrm{m}, \mathrm{n}$ \\
\hline Mimosa bimucronata (DC.) Kuntze & 1 & $\mathrm{E}$ & 0,7 & - & $\mathrm{f}, \mathrm{k}$ \\
\hline Piptadenia gonoacantha (Mart.) J.F. Macbr. & 6 & $\mathrm{E}$ & 4,7 & - & - \\
\hline \multicolumn{6}{|l|}{ Melastomataceae } \\
\hline Miconia sp. Ruiz \& Pav. & 4 & $\mathrm{~F}$ & 0,7 & $\mathrm{e}$ & $\mathrm{n}$ \\
\hline \multicolumn{6}{|l|}{ Meliaceae } \\
\hline Cedrela fissilis Vell. & 3 & $\mathrm{E}$ & 1,7 & $\mathrm{e}, \mathrm{h}$ & - \\
\hline \multicolumn{6}{|l|}{ Moraceae } \\
\hline Morus nigra $\mathrm{L}$. & 7 & $\mathrm{~F}$ & 4 & - & - \\
\hline \multicolumn{6}{|l|}{ Musaceae } \\
\hline Musa paradisiaca $\mathrm{L}$. & 6 & $\mathrm{~F}$ & 6,9 & $\mathrm{n}$ & - \\
\hline \multicolumn{6}{|l|}{ Myrtaceae } \\
\hline Eugenia brasiliensis Lam. & 1 & $\mathrm{~F}$ & 1,7 & & $\mathrm{~d}, \mathrm{e}$ \\
\hline Eugenia uniflora L. & 4 & $\mathrm{~F}$ & 2,2 & $\mathrm{~d}$ & $\mathrm{e}$ \\
\hline Psidium cattleianum Sabine & 1 & $\mathrm{~F}$ & 0,5 & & $\mathrm{~d}, \mathrm{j}, \mathrm{k}, \mathrm{n}$ \\
\hline Psidium guajava $\mathrm{L}$. & 7 & $\mathrm{~F}$ & 3 & $\mathrm{~d}, \mathrm{j}, \mathrm{k}, \mathrm{n}$ & $\mathrm{j}$ \\
\hline Syzygium jambolanum (Lam.) DC. & 9 & $\mathrm{~F}$ & 12,2 & $\mathrm{~d}, \mathrm{~g}, \mathrm{i}, \mathrm{j}$ & - \\
\hline \multicolumn{6}{|l|}{ Rosaceae } \\
\hline Eriobotrya japonica (Thunb.) Lindl. & 1 & $\mathrm{~F}$ & 1,3 & - & - \\
\hline \multicolumn{6}{|l|}{ Rutaceae } \\
\hline Dictyoloma vandellianum A.Juss. & 1 & $\mathrm{E}$ & 0,5 & - & $\mathrm{F}$ \\
\hline Zanthoxylum petiolare A.St.-Hil. \& Tul & 1 & $\mathrm{E}$ & 1,2 & - & $\mathrm{f}, \mathrm{m}$ \\
\hline
\end{tabular}

Referências: (a) Alonso e Langguth (1989) - C. jacchus; (b) Scanlon et al. (1989) - C. jacchus; (c) Passamani (1996) - C. penicillata; (d) Castro et al. (2000) - C. jacchus; (e) Martins e Setz (2000) - C. aurita; (f) Passamani e Rylands (2000) - C. geoffroyi; (g) Miranda e Faria (2001) - C. penicillata; (h) Sant'Anna (2002) - C. penicillata; (i) Vilela e Faria (2002) - C. penicillata; (j) Castro e Araújo (2006) - C. jacchus; (k) Martins (2007) - C. jacchus; (I) Vilela (2007) - C. penicillata; (m) Abbehusen et al. (2007) - C. geoffroyi; (n) Raboy et al. (2008) - C. kuhlii. 


\section{Discussão}

Embora exsudato seja o recurso alimentar que possui maiores registros de consumo em trabalhos realizados com dieta de Callithrix (MARTINS; SETZ, 2000; PASSAMANI; RYLANDS, 2000; CASTRO; ARAÚJO, 2006; RABOY et al., 2008), o forrageio (incluindo a procura e esforços para obtenção) por presa animal é a atividade alimentar registrada predominantemente nos trabalhos de Vilela e Faria (2004), Raboy et al. (2008) e Zago (2012). Da mesma maneira, em ambos os grupos aqui estudados a categoria alimentação/forrageio por presa animal obteve a maioria dos registros, enquanto exsudato obteve os segundos maiores registros, seguido por fruto.

Trabalhos anteriores apontam que proporções de forrageio ou consumo de frutos e exsudatos dependem de condições ambientais que determinam a disponibilidade destes recursos (RYLANDS; FARIA, 1993; VILELA; FARIA, 2004; CASTRO; ARAÚJO, 2006 VILELA, 2007; ZAGO, 2012). Os porcentuais de registros encontrados para cada categoria alimentar amostradas neste trabalho corroboram com estas informações. A disponibilidade de um grande número de árvores que liberam exsudatos que podem ser consumidos a qualquer época do ano e os altos custos de tempo em atividade de escavação do tronco para sua obtenção são fatores que aumentam os registros desta atividade alimentar. Já as árvores de frutos, apesar de numerosas, estiveram com seus recursos amplamente disponíveis apenas em pequenos espaços temporais do ano e exigem menores esforços em sua obtenção, o que resulta numa redução nos registros desta categoria alimentar.

Embora os registros de alimentação suplementar tenham sido os menores valores encontrados, esta categoria alimentar foi bem distribuída ao longo do ano, só não ocorrendo em dois meses dos seis trabalhados com GL e em um mês dos trabalhados com GG. Os pequenos valores encontrados para esta categoria foram influenciados pelo menor esforço e tempo gastos pelos saguis na obtenção do recurso, e pela concentração desta atividade no curto espaço de tempo em que o alimento é disponibilizado. Considerando-se esses menores gastos em esforços e tempo, ainda que com poucos registros, esta categoria alimentar pode possibilitar um grande aporte energético, já que exige gastos calóricos muito pequenos em relação a outras categorias alimentares.

Grandes frequências na disponibilidade de alimentos fornecidos por humanos, já que são recursos alimentares que podem oferecer grande aporte energético, podem ser um fator importante no estabelecimento de primatas em áreas antrópicas e fora de suas áreas de distribuição natural. Os trabalhos de Sant'Anna (2002), Garrido de Paula et al. (2005), Modesto e Bergallo (2008), Zago (2012), Albuquerque e Oliveira (2013) e Silva et al. (2013), realizados com espécies de Callithrix introduzidos (C. penicillata e $C$. jacchus), corroboram com tal afirmação e apontam a alimentação suplementar como um hábito frequente a humanos que habitam ou visitam as áreas habitadas por tais animais. O hábito em alimentar esses primatas pode resultar em aumentos nas suas taxas reprodutivas e densidades populacionais (ZAGO, 2012) o que leva também ao aumento de seus potenciais como espécies invasoras.

Além de favorecer o estabelecimento de espécies de Callithrix fora de suas áreas de ocorrência natural, a alimentação suplementar pode levar a alterações comportamentais e ecológicas nesses animais (BOERE et al., 2000; GARRIDO DE PAULA et al., 2005; MODESTO; BERGALLO, 2008; ZAGO, 2012; SILVA et al., 2013) bem como aumentar a competição dos mesmos com outros primatas. Ruiz-Miranda et al. (2006), em seu trabalho com Leontopithecus rosalia Linnaeus, 1766 (os micos-leões-dourados) reintroduzidos em um programa de conservação da espécie, apontam o aumento da competição entre os micos-leões-dourados em sua distribuição natural e Callithrix spp. introduzidos com a disponibilidade de alimentos suplementares. Garrido de Paula et al. (2005) também argumentam que alimentar Callithrix spp. pode levar a dependência dos mesmos por alimentos suplementares e, ainda, que esta prática pode aumentar o risco de transmissão de zoonoses destes animais a populações humanas.

Dentre as 25 espécies vegetais exploradas por $C$. penicillata neste trabalho, 10 já haviam sido citadas na literatura como presentes na dieta de espécies de Callithrix em sua distribuição natural, assim como outras 10 espécies exploradas já possuíam espécies congêneres 
citadas (Tabela 2). Também pudemos observar que a exploração de exsudatos pelos micos-estrela foi predominantemente realizada em espécies da família Fabaceae, tal resultado é semelhante ao encontrado em trabalhos anteriores. Passamani e Rylands (2000) em trabalho com C. geoffroyi, por exemplo, também encontraram predominância no uso dessa família para exploração de exsudatos. Os mesmos autores encontraram ainda, que os gêneros Inga e Acacia foram os mais utilizados, o que também ocorreu no presente estudo (Tabela 2).

A semelhança encontrada entre as espécies vegetais utilizadas por saguis em sua distribuição natural e neste estudo aponta a disponibilidade de recursos alimentares semelhantes em ambos os locais. A disponibilidade destes alimentos somada a capacidade dos saguis em encontrá-los e consumi-los podem estar relacionados a facilidade dos grupos de $C$. penicillata introduzidos em obter alimentos importantes para sua sobrevivência. A capacidade desses primatas em reconhecer, localizar e consumir esses recursos alimentares pode então, também estar relacionada ao estabelecimento dessa espécie neste novo habitat a que foi exposta.

Altas densidades de uma determinada espécie frutífera e longos períodos de frutificação são características de habitats fragmentados e bordas de floresta que podem determinar a preferência de espécies do gênero Callithrix por estes habitats (RYLANDS, 1996). As espécies mais exploradas para consumo de frutos neste trabalho foram Syzygium jambolanum, Musa paradisiaca e Morus nigra. Os altos registros para a primeira e última espécie parecem relacionarse à abundância de árvores e a alta produtividade de frutos em uma época específica do ano, enquanto para Musa paradisiaca parecem relacionar-se à produção bem distribuída ao longo do ano. Estes padrões de frutificação podem então, ser características deste habitat que reforçam o estabelecimento $C$. penicillata neste fragmento urbano.

As proporções das atividades alimentares encontradas neste trabalho foram semelhantes às encontradas nas distribuições naturais de saguis. Esta informação associada a apontamentos demográficos realizados por Zago et al. (2011) nos permite inferir a aptidão de $C$. penicillata em realizar suas atividades normais de obter alimento neste ambiente a que foi introduzida. A disponibilidade de alimentos fornecidos por humanos, a presença de recursos vegetais semelhantes aos encontrados em distribuição natural e padrões de frutificação vantajosos para C. penicillata foram fatores e características do PECG que possivelmente permitiram a realização normal destas atividades alimentares. Tal inferência corrobora com apontamentos de estudos anteriores (RYLANDS, 1996; SANT'ANNA, 2002; GARRIDO DE PAULA et al., 2005; MODESTO; BERGALLO, 2008; ZAGO; 2012) quanto à flexibilidade ecológica de $C$. penicillata e os fatores que os permitem viver em habitats urbanos e fragmentados.

\section{Agradecimentos}

Agradecemos à administração do Parque Ecológico do Córrego Grande e a Fundação Municipal do Meio Ambiente (FLORAM) por permitirem a coleta de dados, a bolsa fornecida pela Universidade Federal de Santa Catarina a Luciana Zago durante a realização deste trabalho e ao Conselho Nacional de Desenvolvimento Científico e Tecnológico (CNPq) pela bolsa de produtividade a Fernando C. Passos (303757/2012-4).

\section{Referências}

ABBEHUSEN, A.; SILVA, R. M. L.; BARRETO, C. E. Dieta e área de uso do sagüi-da-cara-branca (Callithrix geoffroyi) em Porto Seguro, Bahia. In: BICCA-MARQUES, J. C. (Ed.). A Primatologia no Brasil 10. Porto Alegre: SBPr/EDIPUCRS, 2007. p. 339-351.

ALBULQUERQUE, J. R.; OLIVEIRA, M. A. B. Interações entre humanos e Callithrix jacchus (Linnaeus, 1758) no Parque Estadual Dois Irmãos, Recife-PE, Brasil. In: MIRANDA, J. M. D.; PASSOS, F. C. (Ed.). A Primatologia no Brasil 13. Curitiba: UFPR/SBPr, 2013. (No prelo)

ALONSO, C.; LANGGUTH, A. Ecologia e comportamento de Callithrix jacchus (Primates: Callithrichidae) numa ilha de floresta atlântica. Revista Nordestina de Biologia, João Pessoa, v. 6, n. 2 , p. 105-137, 1989.

ALTMANN, J. Observational study of behavior: sampling methods. Behaviour, Groningen, v. 40, n. 3, p. 227-267, 1974.

BOERE, V.; TILlMANN, L.; RESENDE, M. C.; TOMAZ, C. Uso do espaço e comportamento social em sagüis do Cerrado (Callithrix penicillata) selvagens, no Centro de Primatologia da Universidade de Brasília. In: ALONSO, C.; LANGGUTH, A. (Ed.). A Primatologia no Brasil 7. João Pessoa: Editora da UFRN/ Editora Universitária - UFPB, 2000. p. 35-48. 
CASTRO, C. S. S.; ARAÚJO, A. Diet and feeding behavior of Marmoset Callithrix jacchus. Brazilian Journal of Ecology, São Paulo, v. 7, n. 10, p. 14-19, 2006.

CASTRO, C. S. S.; ARAÚJO, A.; AlHO, C.; FILHO, M. D. Influência da distribuição e disponibilidade dos frutos, na dieta e uso do espaço em Sagüis-do-Nordeste (Callithrix jacchus). In: AlONSO, C.; LANGGUTH, A. (Ed.). A Primatologia no Brasil 7. João Pessoa: Editora da UFRN/Editora Universitária - UFPB, 2000. p. 65-80.

CHIARELlO, A. G.; AGUIAR, L. M. S.; CERQUIERA, R.; MELlO, F. R.; RODRIGUES, F. H. G.; SILVA, V. M. F. Mamíferos ameaçados de extinção no Brasil. In: MACHADO, A.; DRUMMOND, G. M.; PAGLIA, A. P. (Ed.). Livro Vermelho da fauna brasileira ameaçada de extinção. Belo Horizonte: FNMA / Fundação Biodiversitas, 2008. p. 681-874.

COIMBRA-FILHO, A. F. Sistemática, distribuição geográfica e situação atual dos símios brasileiros. Revista Brasileira de Biologia, São Carlos, v. 50, n. 4, p. 1063-1079, 1990.

FERRARI, S. F. Ecological differentiation in the Callitrichidae. In: RYLANDS, A. (Ed.). Marmosets and Tamarins: systematics, behaviour, and ecology. Oxford: Oxford University Press, 1993. p. 315-328.

FERRARI, S. F. Conservation of the Marmosets and Callimicos, In: FORD, S. M.; PORTER, L. M.; DAVIS, L. C. (Ed.). The smallest anthropoids: the Marmoset/Callimico radiation. Series Developments in Primatology: progress and prospects. New York: Springer Press, 2009. p. 465-477.

GARRIDO DE PAULA, H. M.; TÁVORA, R. S.; ALMEIDA, M. V.; PELEGRINI, L. S.; SILVA, G. V.; ZAGANINI, R. L.; LUCINDO, A. Estudos preliminares da presença de saguis no Município de Bauru, São Paulo, Brasil. Neotropical Primates, Arligton, v. 13, n. 3, p. 6-11, 2005.

HESHKOVITZ, P. Living new world monkeys (Platyrrhini). Vol. 1. Chicago: The University of Chicago Press, 1977. 1132 p.

MARROIG, G.; CHEVERUD, J. M. Size as a line of evolutionary resistance: diet and adaptive morphological radiation in new world monkeys. Evolution, New York, v. 59, n. 5, p. 1128-1142, 2005.

MARTINS, I. G. Padrão de atividades do sagüi Callithrix jacchus numa área de Caatinga. 2007. 56 f. Dissertação (Mestrado em Psicobiologia) - Universidade Federal do Rio Grande do Norte, Natal. 2007.

MARTINS, M. M.; SETZ, E. Z. F. Diet of buffy tufted-eared marmosets (Callithrix aurita) in a forest fragment in southeastern Brazil. International Journal of Primatology, New York, v. 21, n. 3, p. 467-476, 2000.

MIRANDA, G. H. B.; FARIA, D. S. Ecological aspects of blackpincelled Marmoset (Callithrix penicillata) in the Cerradão and dense Cerrado of the Brazilian Central Plateau. Brazilian Journal of Biology, São Carlos, v. 61, n. 3, p. 397-404, 2001.

MODESTO, T. C.; Bergallo, H. G. Ambientes diferentes, diferentes gastos do tempo entre atividades: o caso de dois grupos mistos do exótico Callithrix spp. na Ilha Grande, RJ, Brasil. Neotropical Biology and Conservation, v. 3, n. 3, p. 112-118, 2008.

PASSAMANI, M. Uso de árvores gomíferas por Callithrix penicillata no Parque Nacional da Serra do Cipó, MG. Boletim do Museu de Biologia Mello Leitão, Santa Teresa, v. 4, p. 25-31, 1996.
PASSAMANI, M.; RYLANDS, A. B. Home range of a Geoffroy's marmoset group, Callithrix geoffroyi (Primates, Callithrichidae) in South-Eastern Brazil. Revista Brasileira de Biologia, São Carlos, v. 60 , n. 2, p. 275-281, 2000.

RABOY, B. E.; CANALE, G. R.; DIETZ, J. M. Ecology of Callithrix kuhlii and a review of eastern Brazilian marmosets. International Journal of Primatology, New York, v. 29, p. 449467, 2008.

RUIZ-MIRANDA, C. R.; AFFONSO, A. G.; MORAIS, M. M.; VERONA, C. E.; MARTINS, A.; BECK, B. Behavioral and ecological interactions between reintroduced golden lion tamarins (Leontopithecus rosalia Linnaeus, 1766) and introduced marmosets (Callithrix spp., Linnaeus, 1758) in Brazil's Atlantic Coast Forest fragments. Brazilian Archives of Biology and Technology, Curitiba, v. 49, n. 1, p. 99-109, 2006.

RYLANDS, A. B. Habitat and the evolution of social and reproductive behavior in Callitrichidae. American Journal of Primatology, New York, v. 38, p. 5-18, 1996.

RYLANDS, A. B.; COIMBRA-FILHO, A. F.; MITTERMEIER, R. A. Systematics, geographic distribution, and some notes on the conservation status of the Callitrichidae. In: RYLANDS, A. (Ed.). Marmosets and tamarins: systematics, behaviour, and ecology. Oxford: Oxford University Press, 1993. p. 11-77.

RYLANDS, A. B.; FARIA, D. S. Habitats, feeding ecology, and home range size in the genus Callithrix. In: RYLANDS, A. (Ed.). Marmosets and tamarins: systematics, behaviour, and ecology. Oxford: Oxford University Press, 1993. p. 263-272.

SANT'ANNA, F. S. Comportamento alimentar e identificação das árvores produtoras de goma utilizadas por Callithrix penicillata na Ilha de Santa Catarina. 2002. 47 f. Trabalho de Conclusão de Curso (Graduação em Ciências Biológicas) Universidade Federal de Santa Catarina, Florianópolis. 2002.

SANTOS, C.; MORAIS, M. M.; OLIVEIRA, M. M.; MIKICH, S. B.; RUIZ-MIRANDA, C. R.; MOORE, K. P. L. Ecologia, comportamento e manejo de primatas invasores e populaçõesproblema. In: BICCA-MARQUES, J. C. (Ed.). A Primatologia no Brasil 10. Porto Alegre: SBPR/EDIPUCRS, 2007. p. 101-118.

SCANLON, C. E.; CHALMERS, N. R.; MONTEIRO DA CRUZ, M. A. O. Home range use and the exploitation of gum in the Marmoset Callithrix jacchus jacchus. International Journal of Primatology, New York, v. 10, n. 2, p. 123-136, 1989.

SILVA, J. M.; ALBULQUERQUE, J. R.; OlIVEIRA, M. A. B. Alimentos providos por humanos na dieta de Callithrix jacchus. In: MIRANDA, J. M. D.; PASSOS, F. C. (Ed.). A Primatologia no Brasil 13. Curitiba: UFPR/SBPr, 2013. (No prelo)

STEVENSON, M. F.; RYLANDS, A. B. The marmosets, genus Callithrix. In: MITTERMEIER, R. A.; RYLANDS, A. B.; COIMBRA-FILHO, A. F.; FONSECA, G. A. B. (Ed.). Ecology and behavior of Neotropical primates. Vol. 2. Washington: World Wildlife Fund, 1988. p. 131-222.

VILELA, S. L. Simpatria e dieta de Callithrix penicillata (Hershkovitz) (Callitrichidae) e Cebus libidinosus (Spix) (Cebidae) em matas de galeria do Distrito Federal, Brasil. Revista Brasileira de Zoologia, Curitiba, v. 24, n. 3, p. 601-607, 2007.

VILELA, S. L.; FARIA, D. S. Dieta do Callithrix penicillata (Primates, Callithrichidae) em áreas de cerrado no Distrito Federal, Brasil. Neotropical Primates, Arligton, v. 10, n. 1, p. 17-20, 2002. 
VILELA, S. L.; FARIA, D. S. Seasonality of the activity pattern of Callithrix penicillata (Primates, Callitrichidae) in the cerrado (scrub savana vegetation). Brazilian Journal of Biology, São Carlos, v. 64, n. 2, p. 363-370, 2004.

VIVO, M. Taxonomia de Callithrix Erxleben, 1777 (Callitrichidae Primates). Belo Horizonte: Fundação Biodiversitas, 1991. 105 p.

YAMAMOTO, M. E. From dependence to sexual maturity: the behavioural ontogeny of Callitrichidae. In: RYLANDS, A. (Ed.).

Marmosets and Tamarins: systematics, behaviour, and ecology. Oxford: Oxford University Press, 1993. p. 235- 254.

ZAGO, L.; MIRANDA, J. M. D.; SANTOS, C. V.; PASSOS, F. C. Composição e dinâmica de grupos de Callithrix penicillata (É. Geoffroy, 1812) introduzidos em fragmento urbano na Ilha de Santa Catarina. In: MIRANDA, J. M. D.; HIRANO, Z. M. B. (Ed.). A Primatologia no Brasil 12. Curitiba: UFPR/SBPr, 2011. p. 102114.

ZAGO, L. Fatores determinantes no uso do espaço por Callithrix penicillata (E. Geoffroy, 1812) introduzidos em fragmento urbano. 2012. 80 f. Dissertação (Mestrado em Zoologia) Universidade Federal do Paraná, Curitiba. 2012. 